\title{
FORCED CONCENTRATION OSCILLATIONS FOR CATALYTIC REACTIONS WITH STOP-EFFECT
}

\author{
JAN THULLIE \\ Polish Academy of Sciences, Institute of Chemical Engineering, 44-100 Gliwice, ul. Baltycka 5, Poland \\ and \\ ALBERT RENKEN \\ Ecole Polytechnique Fédérale, Institut de génie chimique, EPFL-Ecublens, CH-1015 Lausanne, \\ Switzerland \\ (Received 18 May 1990; accepted 13 June 1990)

\begin{abstract}
The influence of forced concentration oscillations on a catalytic reaction with stop-effect was studied based on two different adsorption-desorption models. Both models predict mean reaction rates which can be more than twice as high as the maximum rate under optimum steady state conditions. An analytical solution is presented to describe the mean performance as a function of concentration, length of period and cycle split.
\end{abstract}

\section{INTRODUCTION}

An interesting phenomenon of rapid increase of reaction rate during deamination of amines on alumina after a stop in the supply of amines was first observed by Hogan (1973). He stopped the diisopropylamine feed into the stream of inert carrier gas in the differential flow reactor and found that the reaction rate of propene formation was increased 2-3 times compared to the steady state value. The rise was only temporary and was followed by a decrease to zero, due to the absence of the reactant in the feed.

This phenomenon was also observed by Koubek et al. $(1980 \mathrm{a}, \mathrm{b})$ for the dehydration of alcohols and the deamination of primary amines on acid-base oxide catalysts. The authors called their observations "StopEffect".

The stop-effect (SE) was explained by two different adsorption-desorption models, suitable to describe the experimentally observed transient behaviour of the catalyst after replacing the reactant by an inert gas. Nowobilski and Takoudis (1986) proposed a model assuming two different active sites, whereby the reactant (amine or alcohol) is strongly adsorbed on an acid site $\left(S_{1}\right)$ of the catalyst and an empty basic site $\left(S_{2}\right)$ is required for reaction to take place. Elementary steps for the model (model I) are:

$$
\begin{gathered}
\mathrm{A}+\overline{\mathrm{S}}_{1} \underset{\mathrm{k}_{-1}}{\stackrel{k_{1}}{\rightleftharpoons}} \mathrm{A} \overline{\mathrm{S}}_{1} \\
\mathrm{~A}+\overline{\mathrm{S}}_{2} \underset{\mathrm{k}_{-2}}{\stackrel{k_{2}}{\rightleftharpoons}} \mathrm{AS} \overline{\mathrm{S}}_{2} \\
\mathrm{~A} \overline{\mathrm{S}}_{1}+\overline{\mathrm{S}}_{2} \stackrel{{ }^{k_{3}}}{\longrightarrow} \mathrm{B}+\mathrm{C}+\overline{\mathrm{S}}_{1}+\overline{\mathrm{S}}_{2} .
\end{gathered}
$$

When the adsorption of $A$ is strong on both sites, active sites $S_{2}$ are blocked by $A$ molecules and the

\footnotetext{
tAuthor to whom correspondence should be addressed.
}

production rate is low in the steady state. After a stop in the flow rate of $A$, it desorbs from the surface. Supposing that the desorption from the basic sites proceeds much faster than from the acid ones $\left(k_{-2} \gg k_{-1}\right)$, the concentration of empty sites is rising quickly, which results in an increase of the reaction rate until the accumulated surface compound $A \bar{S}_{1}$ has been consumed.

A different model was proposed by Koubek et al. (1980a, b) to fit their experimental results (model II). According to model II, the reactant can be adsorbed on an occupied site forming a kind of second layer of A molecules and hence stopping the surface reaction. Model II is summarized as follows:

$$
\begin{gathered}
\mathrm{A}+\underset{\mathrm{S}_{-1}}{\stackrel{k_{1}}{\rightleftharpoons}} \mathrm{A} \overline{\mathrm{S}} \\
\mathrm{A} \overline{\mathrm{S}}+\mathrm{A} \underset{k_{-2}}{\stackrel{k_{2}}{\rightleftharpoons}} \mathrm{A} \overline{\mathrm{S}} \mathrm{A} \\
\mathrm{A} \overline{\mathrm{S}} \stackrel{\boldsymbol{k}_{3}}{\longrightarrow} \mathrm{B}+\mathrm{C}+\overline{\mathrm{S}} .
\end{gathered}
$$

After stopping the reactant feed, the "second layer" blocking the surface will desorb liberating active $A \bar{S}$ species. As a result the reaction rate will increase, going through a maximum and finally tending to zero.

This interesting observation of performance increase under transient conditions leads to the question whether it could be possible to get mean transformation rates higher than in optimum steady state by repeating the feed-interruption regularly and imposing fluctuations to the system concentration in a square wave fashion as indicated in Fig. 1.

\section{MATHEMATICAL MODELLING}

Supposing a differential reactor, the dynamic behaviour of the surface compounds for model $\mathbf{I}$ can be 
described by the following equations:

$\frac{\mathrm{d}\left[\mathrm{A} \overline{\mathrm{S}}_{1}\right]}{\mathrm{d} t}=k_{1}[\mathrm{~A}]\left[\overline{\mathrm{S}}_{1}\right]-k_{-1}\left[\mathrm{~A} \overline{\mathrm{S}}_{1}\right]-k_{3}\left[\mathrm{~A} \overline{\mathrm{S}}_{\mathrm{t}}\right]\left[\overline{\mathrm{S}}_{2}\right]$

$$
\frac{\mathrm{d}\left[\mathrm{AS}_{2}\right]}{\mathrm{d} t}=k_{2}[\mathrm{~A}]\left[\overline{\mathrm{S}}_{2}\right]-k_{-2}\left[\mathrm{~A} \overline{\mathrm{S}}_{2}\right]
$$

where

$$
\left[\overline{\mathrm{S}}_{1}\right]=1-\left[\mathrm{A} \overline{\mathbf{S}}_{1}\right] ;\left[\overline{\mathbf{S}}_{2}\right]=1-\left[\mathrm{A} \overline{\mathbf{S}}_{2}\right] .
$$

Quite similar equations can be derived from model II:

$$
\begin{aligned}
\frac{\mathrm{d}[\mathrm{A} \overline{\mathrm{S}}]}{\mathrm{d} t}= & k_{1}[\mathrm{~A}][\overline{\mathrm{S}}]-k_{1}[\mathrm{~A} \overline{\mathrm{S}}]-k_{3}[\mathrm{~A} \overline{\mathrm{S}}] \\
& -k_{2}[\mathrm{~A}][\mathrm{A} \overline{\mathrm{S}}]+k_{-2}[\mathrm{~A} \overline{\mathrm{S}} \mathrm{A}] \\
\frac{\mathrm{d}[\mathrm{A} \overline{\mathrm{S}} \mathrm{A}]}{\mathrm{d} t}= & k_{2}[\mathrm{~A}][\mathrm{A} \overline{\mathrm{S}}]-k_{-2}[\mathrm{~A} \overline{\mathrm{S}} \mathrm{A}]
\end{aligned}
$$

where

$$
[\overline{\mathrm{S}}]=1-[\mathrm{A} \overline{\mathrm{S}}]-[\mathrm{A} \overline{\mathrm{S}} \mathrm{A}] .
$$

Assuming instantaneous equilibrium for the surface species $\mathrm{AS}_{2}$ and ASA respectively, eqs (2) and (5), the mean reaction rate under forced concentration oscillations can be investigated.

When the reactant concentration changes between zero and $[A]$ the above equations can be reduced to:

$$
\begin{gathered}
\frac{\mathrm{d}[X]_{n}}{\mathrm{~d} t}=-F[X]_{n} \\
\quad \text { for }(n-1) \tau \leqslant t \leqslant(n-1) \tau+\gamma \tau \\
\frac{\mathrm{d}[Y]_{n}}{\mathrm{~d} t}=-(E+F)[Y]_{n}+B \\
\text { for }(n-1) \tau+\gamma \tau \leqslant t \leqslant n \tau \\
n=1,2, \ldots
\end{gathered}
$$

The initial condition is $[X]=D$ for $t=0$, and the continuity condition which follows from eqs (13) and (14) is

$$
\begin{aligned}
& \lim _{t \rightarrow((n-1) \tau+\gamma \tau)}[X]_{n}=\operatorname{Zlim}_{t \rightarrow((n-1) \tau+\gamma \tau}[Y]_{n} \\
& Z \lim _{t \rightarrow n \tau}[Y]_{n}=\lim _{i \rightarrow n \tau}[X]_{n+1} .
\end{aligned}
$$

Notation for model I:

$$
\begin{aligned}
{[X]_{n} } & =\left[\mathrm{A} \bar{S}_{1}\right] \text { for }(n-1) \tau \leqslant t \leqslant(n-1) \tau+\gamma \tau \\
{[Y]_{n} } & =\left[\mathrm{A} \overline{\mathrm{S}}_{1}\right] \quad \text { for }(n-1) \tau+\gamma \tau \leqslant t \leqslant n \tau \\
F & =k_{-1}+k_{3} \\
E & =k_{1}[\mathrm{~A}]-k_{3} K_{41}[\mathrm{~A}] /\left(K_{11}[\mathrm{~A}]+1\right) \\
B & =k_{1}[\mathrm{~A}] \\
D & =B /(E+F) \\
Z & =1 .
\end{aligned}
$$

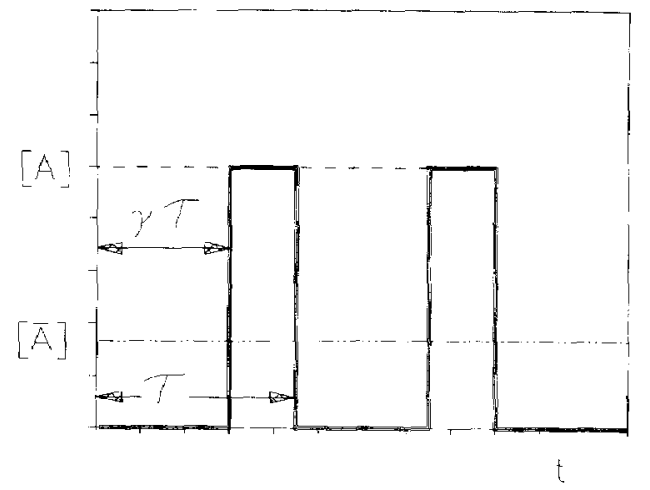

Fig. 1. Concentration of substance A during oscillations.

Notation for model II:

$$
\begin{aligned}
{[X]_{n} } & =[\mathrm{AS}] \quad \text { for }(n-1) \tau \leqslant t \leqslant(n-1) \tau+\gamma \tau \\
{[Y]_{n} } & =[\mathrm{AS}] \quad \text { for }(n-1) \tau+\gamma \tau \leqslant t \leqslant n \tau \\
F & =k_{-1}+k_{3} \\
E & =k_{1}[\mathrm{~A}]\left(K_{11}[\mathrm{~A}]+1\right) \\
B & =k_{1}[\mathrm{~A}] \\
D & =E /(E+F) \\
\mathcal{Z} & =E / B .
\end{aligned}
$$

The solutions of eqs (13) and (14) can be found by a similar way as shown by Thullie et al. (1986). The results are:

$$
\begin{aligned}
{[X]_{n}=} & {\left[D\left(P^{-1} U\right)^{n-1}-D T^{n-1}\left(P^{-1} U T^{-1} S-1\right)\right.} \\
& \left.\times \frac{1-\left(P^{-1} U T^{-1}\right)^{n-1}}{\left.1-P^{-1} U T^{-1}\right]}\right] \exp (-F t) \\
& \text { for }(n-1) \tau \leqslant 1 \leqslant(n-1) \tau+\gamma \tau \\
{[Y]_{n}=} & \frac{B}{E+F}\left[U^{n}-(P T)^{n-1} U(S-1)\right. \\
& \left.\times \frac{\left(1-\left(P^{-1} U T^{-1}\right)^{n-1}\right)}{\left(1-P^{-1} U T^{-1}\right)}-U^{n} S\right] \\
& \times \exp [-(E+F) t]+\frac{B}{E+F} \\
& \quad \text { for }(n-1) \tau+\gamma \tau \leqslant t \leqslant n \tau
\end{aligned}
$$

where

$$
\begin{array}{r}
P=\exp (E \tau) ; T-\exp (F \tau) ; U=\exp \left(E \gamma^{\prime} \tau\right) ; \\
S=\exp (F \gamma \tau) .
\end{array}
$$

Now the mean reaction rate during the $n$th cycle can be obtained. It takes different forms for each model.

MEAN REACTION RATE FROM MODEL 1

The instantaneous reaction rate for this model is defined as:

$$
R_{n}=k_{3}\left[\mathrm{~A} \overline{\mathrm{S}}_{1}\right]\left(1-\left[\mathrm{A}_{2}\right]\right) \text {. }
$$


$\left[\mathrm{AS}_{1}\right]$ is given by eqs (16), (17) and

$$
\left[\overline{\mathrm{S}}_{2}\right]=K_{\mathrm{II}}[\mathrm{A}] /\left(K_{\mathrm{II}}[\mathrm{A}]+1\right) * \mathbf{H}[t-(n-1) \tau-\gamma \tau]
$$

where $\mathbf{H}[t-(n-1) \tau-\gamma \tau]$ is a step function (Heaviside function):

$$
\mathbf{H}[t-(n-1) \tau-\gamma \tau]=\left\{\begin{array}{l}
0 \text { for } t<(n-1) \tau+\gamma \tau \\
1 \text { for } t \geqslant(n-1) \tau+\gamma \tau .
\end{array}\right.
$$

The mean reaction rate over one period is:

$$
\begin{aligned}
\vec{R}_{n}= & \frac{1}{\tau} \int_{(n-1) \tau}^{n \tau} R_{n}(t) \mathrm{d} t=\frac{1}{\tau} \int_{(n-1) \tau}^{(n-1) \tau+y \tau} R_{n}(t) \mathrm{d} t \\
& +\frac{1}{\tau} \int_{(n-1) \tau+y \tau}^{n \tau} R_{n}(t) \mathrm{d} t .
\end{aligned}
$$

After integration one obtains for the $n$th period:

$$
\begin{aligned}
\bar{R}_{n}= & \frac{M}{F \tau}\left[\left(P^{-1} U T^{-1}\right)^{n-1}-\left(P^{-1} U T^{-1} S-1\right)\right. \\
& \left.\times \frac{1-\left(P^{-1} U T^{-1}\right)^{n-1}}{1-P^{-1} U T^{-1}}\right]\left(1-S^{-1}\right) \\
& +\frac{[M-(B-E) D] B(1-\gamma)}{D(E+F)} \\
& +\frac{[M-(B-E) D] B(1-S)}{(E+F) \pi}\left[\left(P^{-1} U T^{-1}\right)^{n-1}\right. \\
& \left.+\frac{1-\left(P^{-1} U T^{-1}\right)^{n-1}}{1-P^{-1} U T^{-1}}\right]\left(S^{-1}-P^{-1} U T^{-1}\right)
\end{aligned}
$$

where

$$
M=k_{3} D \text {. }
$$

When forced cycling is repeated sufficiently often, the results within the periods become identical and one obtains for $n \rightarrow \infty$ :

$$
\begin{aligned}
\lim _{n \rightarrow \infty} \bar{R}_{n}= & \bar{R}=\frac{M}{F \tau} \frac{\left(1-P^{-1} U T^{-1} S\right)}{1-P^{-1} U T^{-1}}\left(1-S^{-1}\right) \\
& +\frac{[M-(B-E) D] B}{D(E+F)}(1-\gamma) \\
& +\frac{[M-(B-E) D]}{(E+F) \tau} \\
& \times(1-S) \frac{\left(S^{-1}-P^{-1} U T^{-1}\right)}{\left(1-P^{-1} U T^{-1}\right)}
\end{aligned}
$$

The mean production rate depends on the cycle split $\gamma$ and the length of period $\tau$. For very long periods, the system will attain the steady state condition within each part of the period. The mean production rate reaches a quasi steady state.

For $\tau \rightarrow \infty$, eq. (23) becomes:

$$
\begin{aligned}
R_{\text {QSS }} & =\frac{[M-(B-E) D] B}{D(E+F)}(1-\gamma] \\
& =k_{3}\left[\mathrm{~A} \overline{\mathrm{S}}_{1}\right]_{S}\left(1-\left[\mathrm{A} \overline{\mathrm{S}}_{2}\right]_{s}\right)(1-\gamma) \\
& =R_{S}(1-\gamma) .
\end{aligned}
$$

For short cycle times a limit of expression (23) can be calculated and the mean reaction rate corresponds to the relaxed steady state:

$$
\begin{aligned}
\lim _{\tau \rightarrow 0} \bar{R}= & R_{R S S}=R_{S}(1-\gamma) \\
& +\frac{[M E+(B-E) D F] \gamma(1-\gamma)}{E(1-\gamma)+F}
\end{aligned}
$$

where $R_{S}$ is the steady state reaction rate obtained for constant concentration [A]. It can easily be proved that the second term in expression (25) is always greater than zero. This result indicates that the mean reaction rate for small cycling times is always greater than in quasi steady state.

Introducing

$$
Q=\frac{M E(E+F)+(B-E) B F}{M E(E+F)-(B-E) B E}
$$

eq. (25) can be rewritten in the form

$$
\boldsymbol{R}_{\boldsymbol{R} S \mathrm{~S}}=\boldsymbol{R}_{\mathrm{S}}\left\{(1-\gamma)+Q \frac{E(1-\gamma)}{E(1-\gamma)+F} \gamma\right\} .
$$

It can be shown that $Q$ is greater than 1 for $E>0$.

As the reaction rate under steady state conditions passes a maximum (Thullie and Renken, 1990), the mean rate uncer periodic operation will be referred to this maximal value. Hence an enhancement factor is defined as:

$$
\psi=\frac{\text { time averaged cyclic production rate }}{\text { maximum steady state production rate }} .
$$

For relaxed steady state operation, the enhancement factor becomes:

$$
\psi=\frac{1}{R_{S_{\text {max }}}}-\lim _{\tau \rightarrow 0} R
$$

The enhancement is shown in Fig. 2 for one set of parameters as a function of concentration of reactant A during the second part of a cycle. It is seen that for an invariant cycle split $(\gamma=$ const), a maximum can occur which is higher than 1 . When an optimal cycle split $\left(y=\gamma_{a p}\right)$ is used. the maximal enhancement attained is up to $12 \%$ over the optimum steady state value for $[\mathbf{A}]=0.1$ (bold line in Fig. 2).

The cnhancement factor increases when both cquilibrium constants $K_{\mathrm{I}}$ and $K_{\text {II }}$ decrease and rate constant $k_{3}$ tends to higher values.

The optimum split is given by:

$$
\begin{aligned}
& \gamma_{o p}=\frac{(E+F)(Q-1)-\sqrt{Q F(E+F)(Q-1)}}{E(Q-1)} \\
& \gamma_{o p}=\frac{(E+F)(Q-1)+\sqrt{Q F(E+F)(Q-1)}}{E(Q-1)} \\
& \text { for } E>0 \\
& \text { for } E<0
\end{aligned}
$$

$$
\text { for } E Q>E+F
$$

$$
\gamma_{o p}=1-\frac{M E}{2 B^{2}} \quad \text { for } E=0 \text { and }[\mathrm{A}]>\frac{k_{3}}{2 k_{1}} \text {. }
$$




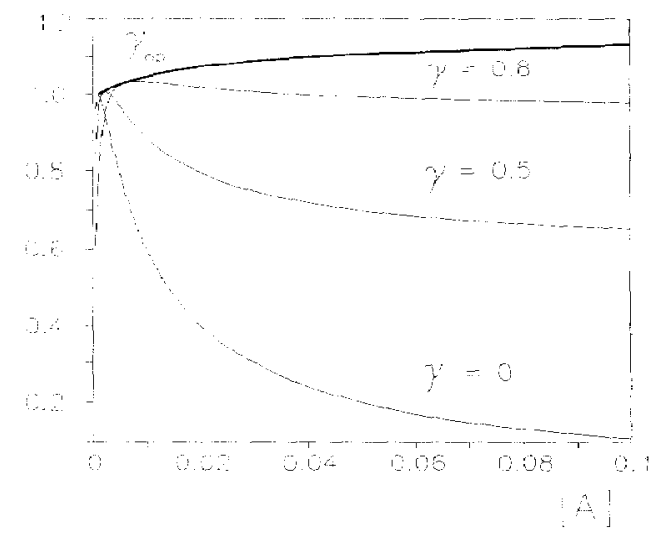

Fig. 2. Enhancement factor as a function of concentration [A] during the second part of a cycle; model I. Input data: $k_{1}$ $=1000 ; k_{-1}=0.001 ; K_{\mathrm{II}}=100 ; k_{3}=0.1$.

Figure 3 shows for the same parameter set the optimum split as a function of reactant concentration $\lceil A\rceil . \gamma_{o p}$ changes quite rapidly for small concentrations $([\mathrm{A}]<0.02)$. For higher concentrations, the value of $\gamma_{o p}$ is greater than 0.9 . This is expected due to the fact that only a short time is required to adsorb enough reactant $A$ on the surface which reacts in the second part of the cycle.

When the equilibrium assumption is not valid, the reaction rate after a stop in the flow of reactant $A$ can be calculated from the relation:

$$
\begin{aligned}
R= & k_{3}\left[\mathrm{~A} \bar{S}_{1}\right]_{S} \exp \left[-\left(k_{-1}+k_{3}\right) t\right. \\
& \left.+\frac{k_{3}}{k_{2}}\left[\mathrm{~A} \bar{S}_{2}\right]_{S}\left(1-\operatorname{cxp}\left(k_{-2} t\right)\right)\right] \\
& \times\left(1-\left[\mathrm{AS}_{2}\right]_{s} \exp \left(-k_{-2} t\right)\right)
\end{aligned}
$$

which is obtained as a solution of an appropriate set of differential equations. Under these conditions the maximum reaction rate is lower compared to that calculated for the equilibrium model. In consequence the mean reaction rate under concentration oscillations will be lower than the value predicted by eq. (25). Some examples of the reaction rate behaviour in contrast to the equilibrium case are given in Fig. 4 .

The time to reach the maximum reaction rate is given as

$$
t^{*}=-\frac{1}{k_{2}} \ln \left[\frac{g}{2 k_{3}\left[\mathrm{AS}_{2}\right]_{s}}\right]
$$

where

$g=k_{-1}+k_{-2}+2 k_{3}-\sqrt{\left(k_{-1}+k_{-2}\right)^{2}+4 k_{3} k_{-2}}$

for

$$
[\mathrm{A}]_{s} \geqslant g / K_{\mathrm{II}}\left(2 k_{3}-g\right) .
$$

If inequality (33) is not satisfied, the increase in the reaction rate after a stop does not take place.

For small time $t^{*}$ corresponding to high values of $k_{-2}\left(k_{-2}>10\right.$, Fig. 4a), the equilibrium assumption

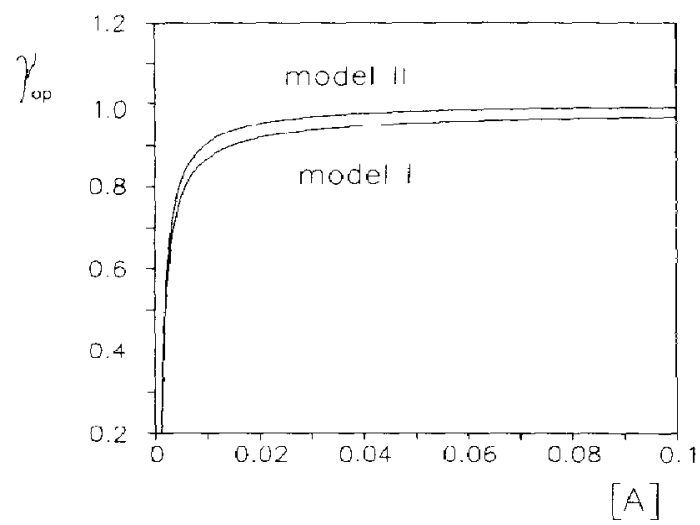

Fig. 3. Optimum split as a function of concentration [A] during the second part of a cycle. Input data: $k_{1}=1000 ; \hat{k}_{-1}$ $=0.001 ; K_{\mathrm{II}}=100 ; k_{3}=0.1$.
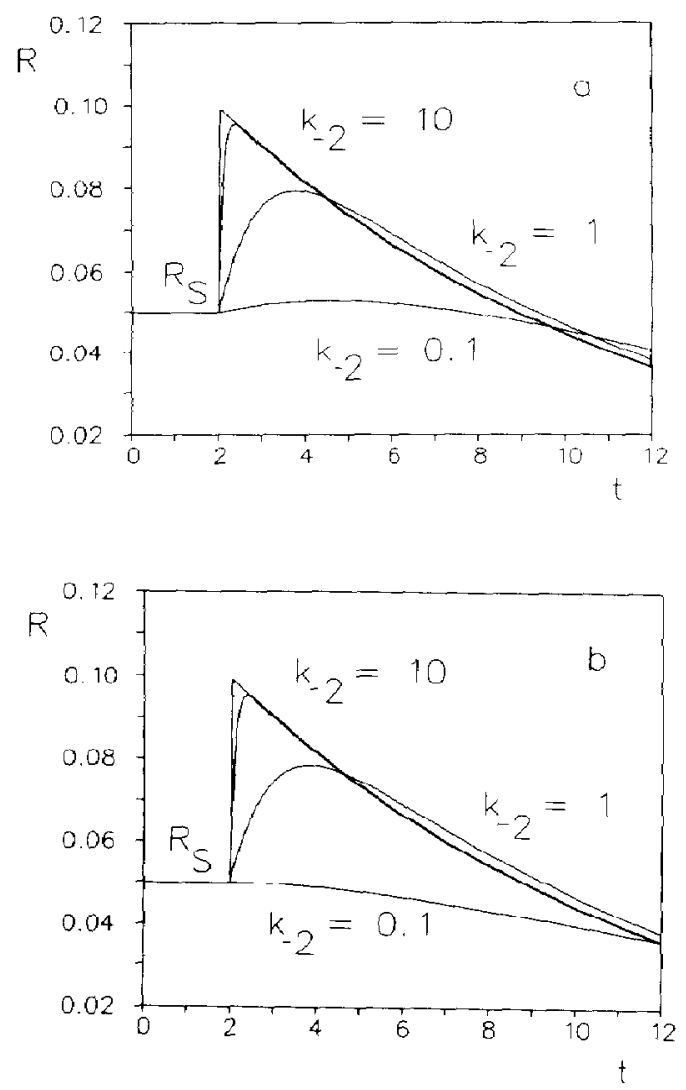

Fig. 4. Reaction rate after a stop in the flow of reactant $A$. Input data: $k_{1}=1000 ; k_{-1}=0.001 ; K_{\mathrm{I}}=100 ; k_{3}=0.1$. (a) Model I. (b) Model III.

can be used and the mean reaction rate is estimated from eq. (23) with cycle times $\tau>t^{*}$.

Equation (25) gives the upper bound of the enhancement attainable for instantaneous adsorption equilibrium. 
MEAN REACTION RATE FOR MODEL II

Based on the kinetic model II, the instantaneous rate of reaction within a period is given by:

$$
R_{n}=k_{3}[\mathrm{AS}] \text {. }
$$

Supposing established equilibrium for the second adsorption step, the time averaged reaction rate can be determined according to eq. (21). After integration one obtains for the $n$th period:

$$
\begin{aligned}
\bar{R}_{n}= & \frac{M}{F \tau}\left[\left(P^{-1} U T^{-1}\right)^{n-1}-\left(P^{-1} U T^{-1} S-1\right)\right. \\
& \left.\times \frac{1-\left(P^{-1} U T^{-1}\right)^{n-1}}{1-P^{-1} U T^{-1}}\right]\left(1-S^{-1}\right) \\
& +\frac{M B}{E}(1-\gamma)+\frac{M B}{E(E+F) \tau} \\
& \times\left[\left(P^{-1} U T^{-1}\right)^{n}-P^{-1} U T^{-1}\right. \\
& \times(S-1) \frac{1-\left(P^{-1} U T^{-1}\right)^{n-1}}{1-P^{-1} U T^{-1}} \\
& \left.-\left(P^{-1} U T^{-1}\right)^{n} S\right]\left(P^{-1} U T^{-1}\right) .
\end{aligned}
$$

When $n \rightarrow \infty \lim _{n \rightarrow \infty} \bar{R}_{n}=\bar{R}$

$$
\begin{aligned}
\bar{R}= & \frac{M}{F \tau}\left[\frac{\left(1-P^{-1} U T^{-1} S\right)\left(1-S^{-1}\right)}{1-P^{-1} U T^{-1}}\right]+\frac{M B}{E}(1-\gamma) \\
& -\frac{M B}{E(E+F) \tau}\left[\frac{\left(1-P^{-1} U T^{-1} S\right)\left(1-S^{-1}\right)}{1-P^{-1} U T^{-1}}\right]
\end{aligned}
$$

For increasing length of period the quasi state is approached and the mean rate is calculated from steady state kinetics:

$$
\lim _{\tau \rightarrow \infty} \bar{R}=\frac{M B}{E}(1-\gamma)=k_{3}[A \bar{S}]_{S}(1-\gamma)=R_{S}(1-\eta)
$$

At very short cycle times $(\tau \rightarrow 0)$, eq. (36) is simplified and a relationship for the relaxed steady state results:

$$
\lim _{\tau \rightarrow 0} \bar{R}=R_{R s s}=R_{S}\left[(1-\gamma)+G \frac{E(1-\gamma) \gamma}{E(1-\gamma)+F}\right]
$$

with

$$
G=\frac{E+F}{B}-\frac{F}{E}>1
$$

The form of eq. (38) is similar to eq. (27) derived for model I. As in the former case, the mean reaction rate for small cycling times is higher compared to quasi steady state values.

For the relaxed steady state an optimum cycle split exists which is dependent on the reactant concentra- tion $[A]$ :

$$
\gamma_{o p}=\frac{(E+F)(G-1)-\sqrt{G F(E+F)(G-1)}}{E(G-1)}
$$

for $E G>E+F$, and $E \geqslant 0$.

As $E$ is always higher or equal to zero, only one relationship is obtained. Optimal split as a function $f[\mathrm{~A}]$ is shown in Fig. 3. Compared to model $\mathrm{I}$ the optimal split is slightly higher for the same concentrations.

The enhancement factor $\psi$ in eq. (28) calculated on the assumption of model II is shown in Fig. 5 and compared to enhancements resulting from model I for optimal cycle split $\gamma_{o p}$. It is interesting to note that the rate at relaxed steady state calculated according to model II $\left(R_{R S S I I}\right)$ is always higher than that from model I $\left(R_{R S S I}\right)$. The relative difference between both models corresponds to about $50 \%$ at $[\mathrm{A}]=0.1$.

As discussed for model $I$, the assumption of instantaneous equilibrium for the second adsorption step results in a maximum enhancement under periodic operation. For non-equilibrium conditions, the reaction rate as a function of time has to be calculated for each period. Due to the fact that the maximum reaction rate after the feed stop is delayed, relaxed steady state is no longer the optimum condition. Nevertheless, mean rates, determined with eq. (36) for high desorption rate constants $\left(k_{-2}>10\right.$, Fig. 4b), give a good approximation for the attainable mean reaction rate. The length of period has to be longer than the time needed for the maximum instantaneous rate after a feed stop. This time can be estimated from the rate-time curve calculated according to eq. (41):

$$
\begin{aligned}
R= & {[\mathrm{A} \bar{S}] \exp \left[-\left(k_{-1}+k_{3}\right) t\right]+\frac{k_{-2}[\mathrm{~A} \overline{\mathrm{SA}}]_{S}}{k_{-1}+k_{3}-k_{-2}} } \\
& \times\left[\exp \left(-k_{-2} t\right)-\exp \left[-\left(k_{-1}+k_{3}\right) t\right]\right]
\end{aligned}
$$

for $k_{-1}+k_{3}-k_{-2} \neq 0$.

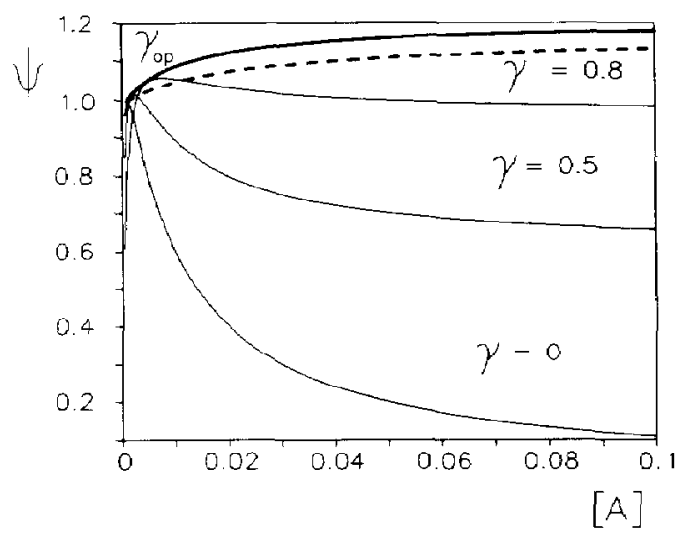

Fig. 5. Enhancement factur as a function of concentration [A] during the second part of a cycle. Input data: $k_{1}=1000$; $k_{-1}=0.001 ; k_{\mathrm{II}}=100 ; k_{3}=0.1$. Solid lines, model II; dashed line, model I. $\boldsymbol{R}_{R S S}\left(\gamma_{o p}\right) / R_{S, \max }$. 


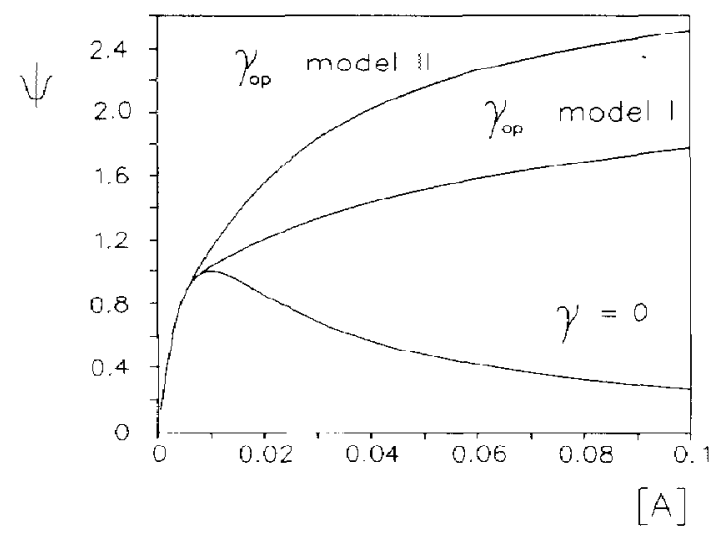

Fig. 6. Comparison of enhancement factors for models I and II. Input data: $k_{1}=10 ; k_{-1}=0.001 ; K_{I I}=100 ; k_{3}=0.1$.

As an example, the transient behaviour is plotted in Fig. 4b. It should be noted that for the same set of parameters, the predicted transient behaviours from both models are hardly distinguishable.

\section{CONCLUSIONS}

The two kinetic models studied in this paper are suitable to describe the experimentally observed sudden incrcase of the reaction rate after a feed stop, called stop-effect (Koubek et al., 1980a, b). The main purpose was to investigate the possibility of getting mean reaction rates under forced concentration oscillations higher than the optimal steady state values. As shown in Fig. 6, the reaction rate under relaxed steady state conditions and applying optimum cycle-split increases monotonically beyond the maximal rate for optimum steady state conditions. The enhancement obtainable depends on the sorption equilibrium constant for the considered adsorption steps. The enhancement factor increases with decreasing adsorption equilibrium constants (Fig. 6) as well as with increasing sorption rates for the second steps, which leads to an adsorption equilibrium. Although both models predict similar steady state and transient reaction rates for identical parameters, there is a remarkable difference for the behaviour under periodic operation. Model II always predicts higher enhancement factors compared to model $\mathbf{I}$. The relative difference can be as high as 50\%, as shown in Fig. 6.

\section{NOTATION}

[A] dimensionless A concentration

[A $\left.\bar{S}_{\mathrm{i}}\right]$ dimensionless $A \bar{S}_{j}$ concentration

$B$ dimensionless constant, $B=k_{1}[\mathrm{~A}]$

$D$ dimensionless constant,

$n=B /(E+F)$ for model $\mathbf{I}$

$D=E /(E+F)$ for model II
$E$ dimensionless constant,

$E=k_{1}[\mathrm{~A}]-k_{3} K_{\mathrm{II}}[\mathrm{A}] /\left(K_{\mathrm{Ir}}[\mathrm{A}]+1\right)$ for model I

$E=k_{1}[\mathrm{~A}]\left(K_{\text {II }}[\mathrm{A}]+1\right)$ for model II

$F \quad$ dimensionless constant, $F=k_{-1}+k_{3}$

$G$ dimensionless constant given by eq. (39)

$g$ dimensionless constant given by eq. (32)

$H \quad$ Heaviside step function

$K_{\mathrm{I}} \quad$ equilibrium constant, $K_{\mathrm{I}}=k_{1} / k_{-1}$

$K_{\text {II }}$ equilibrium constant, $K_{\text {II }}=k_{2} / k_{-2}$

$k_{i} \quad$ dimensionless rate constant

$M \quad$ dimensionless constant, $M=k_{3} D$

$P \quad$ dimensionless constant, $P=\exp (E \tau)$

$Q \quad$ dimensionless constant given by eq. (26)

$R_{S} \quad$ dimensionless steady state reaction rate

$R_{n} \quad$ dimensionless instantaneous reaction rate during $n$th cycle

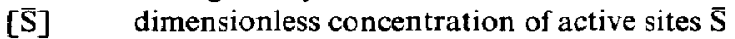

$\left[\overline{\mathbf{S}}_{\mathrm{i}}\right]$ dimensionless concentration of active sites $\overline{\mathbf{S}}_{\mathbf{i}}$

$S \quad$ dimensionless constant, $S=\exp (F \gamma \tau)$

$T \quad$ dimensionless constant, $T=\exp (F \tau)$

$t$ dimensionless time

$t^{*} \quad$ dimensionless time for the maximum reaction rate after a stop in the supply of reactant $A$

$U$ dimensionless constant, $U=\exp (E \gamma \tau)$

$Z$ dimensionless constant,

$Z=1$ for model I

$Z=E / B$ for model II

\section{Greek letters \\ $\gamma \quad$ cycle split \\ $\tau \quad$ cycle time \\ $\psi \quad$ enhancement factor}

\section{Subscripts}

max maximum

op optimum

$S \quad$ steady state

QSS quasi steady state

RSS relaxed steady state

\section{REFERENCES}

Hogan, P., 1973, Ph.D. Thesis, Prague Inst. Chem. Technol., Prague.

Koubek, J., Pasek, J. and Ruzicka, V., 1980a, Exploitation of a nonstationary kinetic phenomenon for the elucidation of surface processes in a catalytic reaction. Proc. 7 th International Congress on Catalysis, Tokyo, 7B, 853.

Koubek, J., Pasek, J. and Ruzicka, V., 1980b, Stationary and nonstationary deactivation of alumina and zeolites in elimination reactions. In Catalyst Deactivation. Elsevier, Amsterdam.

Nowobilski, P. J. and Takoudis, Ch. G., 1986, Periodic operation of chemical reactor systems: are global improvements attainable? Chem. Engng Commun. 40, 249.

Thullie, J. and Renken, A., 1990, Transient behaviour of catalytic reactions with stop-effect. Chem. Engng Commun. (in press)

Thullie, J., Chiao, R. and Rinker, R. G., 1986, Analysis of concentration forcing in heterogeneous catalysis. Chem. Engng Commun. 48, 191. 